\title{
Matheen Ahmed
}

Université de Gand/Fonds de la

Recherche Scientifique-Flandre (FWO)

Achtung Zelig! : deux monstres et un clown au carrefour de l'histoire et de la mémoire médiatique

A chtung Zelig!, qui se déroule pendant la narration à la fois fantastique et absurde pour rendre compte de cette époque terrifiante. Cette BD évoque non seulement les enjeux de la représentation des horreurs à la fois inimaginables et réelles, mais elle exemplifie aussi la présence et le fonctionnement des " mémoires des médias ", c'est-à-dire les mémoires des productions culturelles spécifiques, les œuvres spécifiques ainsi que les médias et les arts de la scène (cirque, théâtre). Cet article a pour but de s'interroger sur le fonctionnement, surtout la mise en œuvre des connotations, des principales mémoires médiatiques dans Achtung Zelig!, à savoir le carnavalesque, Maus d'Art Spiegelman, Mickey au Camp de Gurs de Horst Rosenthal et Le Dictateur de Charlie Chaplin.

La médiation de l'Holocauste à travers monstres, clowns et chatons

Dessinée par Krzysztof Gawronkiewicz et illustrée par Krystian Rosenberg, Achtung Zelig! est parue en polonais en 2004. Sa traduction française a été publiée un an plus tard chez Casterman. Selon la postface de Grzegorz Rosiński (artiste polonais connu pour sa série Thorgal), les 
premières pages de cette $B D$ ont été publiées dans un fanzine des années 1990. Pour Rosiński, l'humour déployé dans Achtung Zelig! pour raconter la Grande Guerre rend cette œuvre comparable à La Vita è bella, un film de Robert Benigni sur la Seconde Guerre mondiale qui recourt à la comédie pour raconter une histoire tragique ${ }^{1}$. Par conséquent Achtung Zelig! se situe dans la sphère des narrations non conventionnelles à propos de l'Holocauste, dans lesquelles le rire et l'absurdité sont mobilisés pour protester contre l'horreur.

Lorsqu'il s'agit de représenter le traumatisme, le réel est problématique, car, face à la violence extrême, la raison s'effondre. Les productions culturelles consacrées à un traumatisme collectif peuvent soulever des problèmes éthiques, car elles ont tendance à esthétiser l'évènement et à suivre les codes du divertissement, risquant ainsi de dissimuler une réalité dure. En optant pour la BD pour narrer l'Holocauste, Art Spiegelman " réinsère, d'une manière hérétique, l'Holocauste dans la sphère politique en mettant l'accent sur son imbrication inévitable dans la sphère publique ainsi que dans la production des marchandises ${ }^{2}$. En d'autres termes, l'engagement politique d'aujourd'hui doit satisfaire les lois du marché, fondées sur la nécessité d'attirer des lecteurs, de les divertir et potentiellement de les toucher. Ainsi les différences entre la mémoire traumatique et le divertissement dans les productions culturelles traitant d'évènements traumatiques deviennent-elles floues ${ }^{3}$. Selon Rotheberg, " l'espace BD-esque » de Maus est " extrêmement mé-

\footnotetext{
${ }^{1}$ G. Rosiński, Postface, [dans :] K. Gawronkiewicz, K. Rosenberg, Achtung Zelig!, W. Kasprzak (trad.), Brussels, Casterman, 2004, p. 55. Désormais abrégé en $A Z$.

2 M. Rothberg, " "We Were Talking Jewish": Art Spiegelman's Maus as "Holocaust" Production ", [dans :] Contemporary Literature, 1994, n 4, p. 671 , trad. M.A.
}

3 A. Huyssen, Present Pasts: Urban Palimpsests and the Politics of Memory, Stanford, Stanford University Press, 2003. 
diatisé $»^{4}$ (il implique diverses couches médiatiques). II " capture » de cette façon " l'hyperintensité d'Auschwitz à la fois plus réel que le réel et plus impossible que l'impossible ${ }^{5}$. Cet aspect de médiatisation permet de comprendre les mécanismes de référentialité déclenchés par Achtung Zelig!. L'espace " extrêmement médiatisé " de la BD, exploité de plus en plus par les romans graphiques traitant de la mémoire personnelle et collective (au lieu des genres récurrents de I'humour, de la fantaisie et du super-héros), peut apporter un nouvel éclairage sur la manière dont la BD fonctionne, en particulier la façon dont ses cases s'emparent d'un réseau vaste de références médiatiques. Comme l'affirment Astrid Erll et Ann Rigney, " la dynamique de la mémoire culturelle [...] est étroitement liée aux processus de remédiation ${ }^{6}$.

Bien que le concept de mémoire médiatique ne soit pas inconnu7, il est assez rarement utilisé, malgré le couplage de deux notions clés actuelles : le médium et la mémoire. Ce concept met l'accent sur le prolongement des mémoires collectives et personnelles à travers des médias. II inclut les mémoires médiatiques plus larges, telles que les modes et les conventions génériques, ainsi que les mémoires médiatiques spécifiques qui continuent de résonner dans les productions culturelles récentes. La mémoire médiatique est un concept particulièrement utile pour l'étude de la BD parce que le mélange des éléments textuels et visuels présents dans la plupart des BD leur permet d'incorporer des allusions aux autres médias qui

\footnotetext{
${ }^{4}$ M. Rothberg, " "We Were Talking Jewish": Art Spiegelman's Maus as "Holocaust" Production ", op. cit., p. 671, trad. M.A.

5 Ibidem.

${ }^{6}$ A. Erll, A. Rigney, "Introduction: Cultural Memory and its Dynamics ", [dans :] Idem (dir.), Mediation, Remediation and the Dynamics of Cultural Memory, Berlin, Walter de Gruyter, 2009, p. 5, trad. M.A.

7 Cf. M. Neiger et al., (dir.), On Media Memory. Collective Memory in a New Media Age, Basingstoke, Palgrave Macmillan, 2011 ; J. Van Dijck, Mediated Memories in the Digital Age, Stanford, Stanford University Press, 2007.
} 
se basent sur l'image et/ou le texte, ainsi qu'aux autres productions culturelles (comme les arts de la scène tels que le cirque et la comédie burlesque). Pour Thierry Smolderen, la BD est " polygraphique " $^{8}$, I'homologue visuel de la polyphonie dans les œuvres littéraires décrites par Bakhtine. Capable d'incorporer plusieurs styles qui peuvent être visuels et textuels, elle se base sur une constellation d'allusions à divers systèmes de représentation, allant de l'art académique au graffiti. Cette essence polygraphique facilite l'inclusion d'une grande variété de mémoires médiatiques. En outre, elle permet à la BD de se régénérer constamment, d'attirer et de divertir ses lecteurs.

En incorporant et en transmettant ces diverses mémoires, la BD participe également à la double logique de remédiation décrite par Jay $D$. Bolter et Richard Grusin. Dans leur ouvrage phare, ceux-ci montrent comment les médias contemporains aspirent à effacer toute trace de médiation - ce qu'ils appellent le processus d'immédiateté - tout en combinant plusieurs médias - ce qu'ils appellent le processus d'hypermédiateté. "Ce qui est nouveau dans les nouveaux médias ", selon Bolter et Grusin, " c'est la manière particulière dont ils remanient des médias plus anciens et la manière dont les médias plus anciens se remanient eux-mêmes pour répondre aux défis des nouveaux médias $1{ }^{9}$. Illustrant de nouvelles tendances dans le médium relativement ancien de la BD (ancien car la page imprimée reste son support préféré), Achtung Zelig! vise l'hypermédiateté en étant dépendant des mémoires médiatiques suivantes : d'abord le carnavalesque; ensuite l'usage des animaux dans les BD sur I'Holocauste, exemplifié par Maus et Mickey au Camp de Gurs ; enfin Le Dictateur de Charlie Chaplin, qui s'intègre

8 T. Smolderen, Naissance de la bande dessinée : de William Hogarth à Winsor McCay, Bruxelles, Les Impressions Nouvelles, 2009, p. 5.

9 J. Bolter, R. Grusin, Remediation: Understanding New Media, Cambridge, MIT Press, 2000, p. 15, trad. M.A. 
à un réseau rhizomatique de références culturelles, la plus importante étant la pratique transmédiale et moderne de la comédie burlesque.

La $\mathrm{BD}$, le cinéma chaplinesque ainsi que les caractéristiques récurrentes du divertissement populaire, comme les personnages, les gestes et les costumes carnavalesques du cirque et de la pantomime, persistent comme mémoires médiatiques qui véhiculent une vaste gamme de connotations dans le narratif nonconventionnel de I'Holocauste d'Achtung Zelig!. À la fois transculturelles et transgénérationnelles, ces mémoires reflètent la tendance récente à conceptualiser la mémoire comme un espace transversal, qui s'affranchit des frontières classiques (entre les médias, les générations et les cultures) ${ }^{10}$.

Nous commencerons par situer Achtung Zelig! dans un espace carnavalesque désorientant et peuplé de corps fantastiques, en d'autres mots un espace qui bouleverse la rationalité. Ensuite nous étudierons les ressorts des mémoires médiatiques de Maus, de Mickey au Camp de Gurs ainsi que du film Le Dictateur.

Vestiges du carnavalesque : espaces liminaux et êtres autrifiés

Achtung Zelig! utilise un style hybride où les personnages grotesques se trouvent dans un espace liminal et flou qui représente la campagne automnale d'une Pologne envahie par les nazis ${ }^{11}$. Le contexte historique de cette BD est bien connu par le lecteur contemporain. Pourtant cette époque cauchemardesque de la Deuxième Guerre mondiale est narrée d'une manière

\footnotetext{
${ }^{10} \mathrm{Cf}$. L. Bond et al., Memory on the Move: Tracing the Dynamics of Memory Studies, New York, Berghahn, 2017.

${ }^{11}$ Voir la contribution de Wojciech Birek sur le style de Gawronkiewicz, qu'il décrit, dans le cas d'Achtung Zelig! comme conceptuel : W. Birek, " Grafische Stilistik der Comics Krzysztof Gawronkiewiczs. Versuch einer Charakterisierung ", [dans :] K. Kupczynska, R. Makarska (dir.), Comic in Polen/Polen im Comic, Berlin, Christian A. Bachmann, 2016, p. 166.
} 
audacieusement absurde, ancrée dans le contraste entre la représentation onirique du paysage et la représentation vraisemblable des corps monstrueux. L'espace onirique évoque l'inconscient où se trouve le traumatisme non résolu. En même temps cet espace où la réalité est temporairement suspendue, où l'impossible devient possible, est aussi carnavalesque : les corps grotesques, toujours amorphes, évoquent cette esthétique subversive.

Exemplifiant les inclinaisons carnavalesques de la BD, l'officier nazi que l'on rencontre est un nain qui a autrefois joué dans un cirque viennois avec son propre numéro intitulé "Émile et les Chenapans " $(A Z, 12)$. Quand la guerre a commencé, tous les cirques ont été incorporés dans l'armée, mais, à cause de sa petite taille, le rêve d’Émile de devenir pilote n'a pas pu se réaliser. Les similarités avec la vie d'Hitler, qui a essayé sans succès de devenir un artiste à Vienne, sont évidentes. Le mode humoristique du récit rappelle d'autres satires sur Hitler, surtout Le Dictateur de Chaplin, mais aussi La Résistible Ascension d'Arturo Ui de Bertold Brecht (écrite en 1941, parue en 1959), où le personnage principal est composé en référence à l'histoire et aux traits d'Hitler et du mafioso Al Capone. Dans Achtung Zelig!, il s'agit plutôt d'allusions qui se distinguent des transpositions directes. Émile, par exemple, n'est pas une caricature fidèle d'Hitler, car il est subordonné aux autres officiers (même si nous ne rencontrons jamais ses supérieurs). Cette caricature infidèle incite le lecteur à examiner et réexaminer les liens entre l'absurde fantaisie de la BD et la réalité historique. De plus, l'exagération de la folie d'Émile, le presquedoppelgänger hitlérien, réduit son pouvoir (et par extension I'horreur de son homologue historique). L'ambiguïté de ce personnage influence le rire qu'il provoque, qui ressemble à son tour au rire carnavalesque ${ }^{12}$.

12 M. Bakhtin, Rabelais and His World, H. Iswolsky (trad.), Bloomington, Indiana University Press, 1984, p. 20. 
Sur le mode absurde qui imprègne l'ensemble de la $\mathrm{BD}$, Émile parle de ses aspirations et de sa solitude pendant la guerre avec les deux juifs qu'il devrait traquer. Les corps anthropomorphiques du père et du fils sont éclipsés par leurs visages monstrueux : Zelig Jr. a la tête d'une grenouille tandis que Zelig Sr. a le visage d'un extraterrestre. Pour Émile, l'apparence monstrueuse de la famille Zelig ouvre la possibilité d'une amitié avec ces deux êtres qui sont aussi des " autres", anormaux comme lui-même. En voyant les bandes avec l'étoile de David autour des bras des deux Zelig, Émile présume qu'elles font partie de leur déguisement $(A Z, 11)$. La réalité des deux personnages est plus dure non seulement parce qu'ils sont en train de fuir la guerre, mais aussi parce qu'ils sont ostracisés par la communauté juive dont, officiellement, ils sont membres :

Nous avions toujours vécu à l'écart du village. Les juifs du village ne nous considéraient pas comme étant des leurs, bien que notre famille ait vécu là depuis des générations. Notre apparence n'y est pas pour rien. $(A Z, 7)$

Les Zelig habitaient à l'écart du village, dans l'espace liminal du bois où toute l'histoire de la BD se déroule. Cet espace reste imprévisible, tout comme Émile qui (comme Hitler) est susceptible de sautes d'humeur. La tension est donc toujours présente dans l'histoire car l'équilibre délicat de la paix peut à tout moment céder au profit de la violence.

L'arrestation des Zelig a lieu un peu plus tard, à la suite d'une bagarre provoquée par un chaton que, lors de leurs adieux à Émile, ce dernier offre en cadeau au petit Zelig (Fig. 1). Tandis que le garçon est ravi, son père est furieux et lui ordonne de le rendre. Il s'avère qu'Émile avait reçu l'ordre d'arrêter les chats, qui devaient servir pour des expériences indicibles - un tampon avec le mot " censuré " masque les mots d'Émile - et être remplacés par les chats du Reich. Ceci rappelle - en le dénigrant mais aussi en réduisant sa gravité - le Generalplan Ost du 
Troisième Reich, qui visait à éliminer les populations " nonaryennes " d'Europe de l'Est et à les remplacer par une population allemande. Dans un acte à la fois héroïque et - au vu des atrocités commises sous le régime nazi irréaliste, Zelig $\mathrm{Sr}$ libère les chats qui échappent aux maladroits soldats nazis. Furieux et déçu par les Zelig, Émile les enferme dans le wagon où les chats avaient été emprisonnés et se met en route pour les livrer aux maîtres de torture $(A Z, 27)$.

Malgré ces événements sombres, Achtung Zelig! a une fin plutôt optimiste. Les chats libérés avertissent les partisans polonais cachés dans le bois. Déguisés en félins, un caporal et un lieutenant se laissent capturer et enfermer dans le même wagon que les Zelig. Ce déguisement renforce l'atmosphère carnavalesque de la BD dans laquelle tous les personnages, avec leur apparence grotesque et leur costume, mettent l'accent sur leur essence performative, non seulement au sens de performance pour créer un spectacle mais aussi au sens de la performance sociale comme décrite par la philosophe Judith Butler, mais qui dans le contexte de cet article va au-delà de la performance des genres pour signifier la performance de l'identité ${ }^{13}$. Une fois que le convoi allemand se trouve dans les profondeurs du bois, il est vaincu par l'armée des partisans.

La période du carnaval marque le moment où toutes les coutumes, conventions et hiérarchies sociales sont mises de côté et où de nouvelles identités peuvent être adoptées. La subversion des pratiques carnavalesques réside dans la mise en exergue du corps et des fonctions corporelles comme décrite par Bakhtin qui parle d'une matérialité corporelle qui est grotesque ou exagérée) ${ }^{14}$. Ici, comme dans Achtung Zelig!, il s'agit d'une perfor-

${ }^{13}$ Cf. J. Butler, Gender Trouble: Feminism and the Subversion of Identity, London, New York, Routledge, 1990.

${ }^{14}$ Cf. M. Bakhtin, Rabelais and His World, op. cit. 
mance corporelle qui transcende les normes sociaux. Le monstre et le clown sont également des types anormaux. Elle réside dans le rire irrévérencieux, qui dégrade sa cible en soulignant sa matérialité et sa corporalité. Si l'on tient compte de la malléabilité des corps et des espaces, un phénomène assez courant dans la $\mathrm{BD}^{15}$, la présence du carnavalesque - une performance qui est vécue et anormale, si ce n'est que pour une durée limitée a une dimension autoréflexive dans Achtung Zelig! où les personnages principaux incarnent la performativité ou la performance d'une identité. L'identité elle-même devient interchangeable avec un rôle ou les types auxquels ils appartiennent : le clown pour Émile et le monstre pour les Zelig.

Bien qu'ils représentent les survivants de l'Holocauste d'une manière non conventionnelle et même polémique, les Zelig renvoient à des types courants dans les BD, comme les héros et les méchants. Les monstres euxmêmes incarnent une mémoire spécifique et corporelle de la BD qui est leur demeure par excellence ${ }^{16}$. Les Zelig illustrent non seulement les sept thèses sur la monstruosité de Jeffrey Jerome Cohen ${ }^{17}$, mais aussi la catégorie esthétique de l'intéressant décrite par Sianne Ngai. Selon elle, l'intéressant est " étroitement lié à la perception de la différence ou de la nouveauté dans le contexte de

15 Voir, par exemple, S. Bukatman, Poetics of Slumberland: Animated Spirits and the Animating Spirit, Berkley, University of California Press, 2012, p. 20.

${ }^{16}$ Voir, par exemple, les séries d'horreur publiées par l'éditeur américain EC Comics avant la censure (c'est-à-dire avant le Comics Code de 1954), qui ont connu un grand succès. Pour la persistance des monstres dans la $\mathrm{BD}$ anglo-saxonne contemporaine, voir le livre de Julia Round: Gothic in Comics and Graphic Novels : A Critical Approach, Jefferson, McFarland, 2014. Même si les BDs francophones sont moins peuplées par les monstres horrifiques, les corps déformés (et donc monstrueux) persistent dans la plupart des BDs.

17 J. Cohen, "Monster Culture (Seven Theses) ", [dans :] Idem (dir.), Monster Theory: Reading Culture, Minneapolis, University of Minnesota Press, 1996, p. 3-25. 
l'attendu et du familier ${ }^{18}$. En d'autres mots, l'intéressant est une catégorie qui met en valeur le potentiel de I'hybridité. "Le monstre ", d'après la quatrième thèse de Cohen, " réside au seuil de la différence " $^{19}$. Les Zelig incarnent cette différence, car ils ne sont même pas acceptés par leur communauté.

Le costume d'Émile, composé d'un bonnet long, pointu et parsemé de petites swastikas, et d'une cape garnie de fourrure est aussi ridicule que son titre officiel : magicien-pisteur. La performativité de sa profession précédente de clown est conservée mais dépouillée de toute trace de comique. Malgré leur maladresse et leur innocuité (au moins superficielle), les clowns modernes connaissent bien la violence, comme le montre AnnaSophie Jürgens qui trace une généalogie du Joker (l'ennemi juré de Batman) en commençant par la création du personnage de Pierrot par le mime Jean-Gaspard Debureau au XIXe siècle ${ }^{20}$. Dans son étude de la convergence de l'horreur et de la comédie, Noël Carroll souligne la monstruosité de tous les clowns ${ }^{21}$. Malgré son apparence ridicule, la nature néfaste d’Émile ne peut pas être sous-estimée : des têtes de mort sont reflétées dans ses lunettes quand les Zelig se font capturer au début de l'histoire $(A Z, 9)$, et sa rage extrême, après qu'il a été frappé par Zelig Jr. quand son père a libéré les chats, est exprimée par deux pages noires sur lesquelles apparaissent son visage furieux et les mots " Tu m'as démoli l'arcade sourcilière !!! » en rouge $(A Z, 24-25)$.

Selon Noël Carroll, les racines de la combinaison de l'horreur et de l'humour sont à trouver dans les

\footnotetext{
18 S. Ngai, Our Aesthetic Categories: Zany, Cute, Interesting, Cambridge, Harvard University Press, 2012, p. 120, trad. M.A.

19 J. Cohen, "Monster Culture (Seven Theses) ", op. cit., p. 7, trad. M.A.

${ }^{20}$ Cf. A.-S. Jürgens, "The Joker: A Neo-Modern Clown of Violence ", [dans :] Journal of Graphic Novels and Comics, 2014, n 4, p. 441-454.

${ }^{21}$ N. Carroll, "Horror and Humor ", [dans :] The Journal of Aesthetic and Art Criticism, 1999, n² 2, p. 155.
} 
interprétations différentes des mouvements mécaniques: tandis que pour Freud l'automate était une source de l'étrange (unheimlich), Bergson a localisé l'essence du rire dans l'observation du fait que "l'humanité était incrustée dans le mécanique ${ }^{22}$. L'humour étant fondé sur l'incongruité, "le rapprochement des idées disparates ou contrastées ", la transformation du clown en un monstre et vice versa est rendue possible ${ }^{23}$. Ceci explique comment les êtres autrifiés (transformés en " autres ») dans Achtung Zelig! échangent, avec une aisance carnavalesque, les connotations d'humour et d'horreur qui leur sont associées : le clown est malfaisant et les monstres sont gentils. Les mémoires médiatiques des deux types de personnages - du cirque et du genre de l'horreur dans les médias visuels - soulignent l'horreur insaisissable et l'absurdité de l'histoire.

Sur la dernière page de la BD nous apprenons que cette histoire est seulement le début du voyage du père et du fils et que le pire est encore à venir, mais en réalité Achtung Zelig! reste sans suite. C'est aussi à la fin que Zelig Jr, qui narre cette histoire rétrospectivement, constate l'impossibilité de distinguer la fiction de la réalité, et l'entrelacement inévitable de l'imagination et de la mémoire $(A Z, 54)$. Au lieu de représenter la réalité, cette $\mathrm{BD}$ fonctionne à travers les mémoires médiatiques du carnavalesque et les productions culturelles - surtout les BD et les films - sur l'Holocauste.

Les mémoires médiatiques de Maus, Mickey Mouse et Charlie Chaplin

Maus, le roman graphique iconique d'Art Spiegelman publié entre 1980 et 1991, n'est jamais mentionné dans Achtung Zelig!. Mais la présence des chats, des êtres

22 Ibidem, p. 146, trad. M.A.

${ }^{23}$ Ibidem, p. 153, trad. M.A. 
anthropomorphiques avec des corps humains dans une BD sur l'Holocauste qui implique un père juif et son fils, évoque cette œuvre, malgré les grandes différences entre les deux BD. Maus est une histoire autobiographique narrée, au-delà de la métaphore animalière, sur un mode assez réaliste. Achtung Zelig!, par contre, entremêle le fantastique et l'absurde dans une mesure rare pour les narrations sur l'Holocauste. " Ni le fantastique, ni la BD, et certainement pas les deux combinés, ne doivent être négligés ou sous-estimés dans leur potentiel à interroger, examiner et expliquer les aspects de l'histoire ${ }^{24}$, conclut Glyn Morgan dans son article sur le rôle du fantastique pour visualiser l'Holocauste. Nous examinerons ici de plus près le fonctionnement du fantastique et de l'absurde dans Achtung Zelig! en commençant par l'effet, tout à fait brechtien, de la distanciation des deux modes de narration.

Tandis que Maus se base sur des faits autobiographiques, ce qui rend le contraste entre le réel et la BD encore plus discordant, Achtung Zelig!, n'ayant pas cet aspect autobiographique, adopte une narration ludique qui rassemble des personnages improbables et absurdes dans un cadre fantastique. Contrairement aux protestations et aux restrictions suscitées par la série Hitler=SS des années 1980, qui était ouvertement caricaturale, Achtung Zelig!, une des rares BD qui racontent l'Holocauste de manière humoristique, a été accueillie de manière très positive ${ }^{25}$. La BD a été louée pour son humour absurde mais stimulant et la poésie sombre qui imprègnent ses pages. Selon Johnathan Haudot, cet accueil favorable est dû à l'absence de caricature. En effet, selon un lecteur de la BD (propos

${ }^{24}$ G. Morgan, "Speaking the Unspeakable and Seeing the Unseeable: The Role of Fantastika in Visualising the Holocaust, or, More than just Maus ", [dans :] The Luminary, 2015, n 6, www.lancaster.ac.uk/luminary/ issue6/issue6article3.htm, trad. M.A.

25 J. Haudot, Shoah et bande dessinée, Paris, L'Harmattan, 2012, p. 166. 
recueillis par Jonathan Haudot), l'absurdité, exemplifiée par les corps monstrueux et le clown, rend la BD plus proche du Rhinocéros d'Eugène lonesco que de Maus, et lui permet d'associer l'humour et l'Holocauste ${ }^{26}$. Cette association délicate est rendue possible par le côté provoquant de l'humour absurde, ainsi que par la distance critique et mystifiante (car l'histoire réelle n'est jamais racontée) du contenu historique. En outre, l'élément comique reste dominé par la menace d'une violence immédiate, tout comme le passé clownesque d'Émile et son apparence ridicule sont amoindris par son pouvoir violent.

La capacité d'Achtung Zelig! à susciter l'empathie couplée à la distance critique illustre le terme de " déséquilibre empathique ${ }^{27}$ (empathic unsettlement) introduit par Dominick LaCapra. Cet effet et la combinaison de l'absurdité et de la fantaisie rappellent Mickey au camp de Gurs (1942), petite BD ou livre illustré de Horst Rosenthal, décédé la même année à Auschwitz. "Publié sans l'autorisation de Walt Disney ", comme annoncé sur sa couverture, la BD spécule sur ce qui aurait pu arriver si Mickey Mouse était allé se promener en France pendant l'Occupation. Incapable de fournir les papiers d'identité demandés par un officier, Mickey se retrouve à Gurs, un camp d'internement où les ressortissants non français étaient détenus. Les conditions de vie dans le camp sont atroces; Mickey les raconte d'une manière directe, simple, mais aussi humoristique. Quand il n'arrive plus à supporter la vie dans le camp, Mickey, en affirmant son essence fictionnelle, s'efface et se met en route pour les États-Unis emblématisés par I'horizon newyorkais. Cette fin heureuse est gâchée par le fait que, comme des milliers de juifs, Rosenthal, n'ayant

${ }^{26}$ Ibidem, p. 96-97.

27 Cf. D. LaCapra, Writing Trauma, Writing History, Baltimore, John Hopkins University Press, 2000. 
jamais réussi à obtenir un visa pour les États-Unis, ne pouvait pas échapper aux horreurs de la guerre ${ }^{28}$. La fin est ainsi comparable à celle d'Achtung Zelig!, qui est également assombrie par la réalité historique de I'Holocauste.

Soulevant les similarités entre les premières manifestations de Mickey Mouse et de Chaplin, l'écrivain John Updike les considère comme des personnages énergétiques exprimant " nos fantasmes d'agilité infinie [et] d'apesanteur parfaite ${ }^{29}$. Mickey et Chaplin sont des créations modernes exemplifiant les corps incroyablement résistants qui peuplent la $B D$ depuis ses débuts ${ }^{30}$. Nous allons examiner la mémoire médiatique des films de Charlie Chaplin pour montrer comment elle renforce les couches subversives de Achtung Zelig!.

La seule référence directe à Chaplin se trouve dans un phylactère de Zelig Jr. qui exprime les pensées du petit garçon pendant qu'Émile essaie d'expliquer pourquoi il a capturé les chats $(A Z, 19$, Fig. 2$)$. Ce phylactère contient exclusivement des images d'un épisode burlesque du film Le Dictateur, dans lequel Chaplin joue le rôle d'Hitler (Adenoid Hynkel) et d'un barbier juif qui lui ressemble. Ayant perdu la mémoire pendant la Grande Guerre, le barbier s'échappe de l'hôpital militaire quand Hynkel est déjà au pouvoir. Par conséquent, il n'arrive pas à comprendre la persécution des juifs allemands par les structures censées protéger tous les citoyens. Dans le phylactère de Zelig Jr., le dictateur est en train d'avoir une de ses crises et il a adopté la position d'un animal à quatre pattes. Le petit recourt donc aux images du film de

28 J. Kotek, D. Pasamonik, Mickey à Gurs: Les carnets de dessin de Horst Rosenthal, Paris, Calmann-Lévy, 2014, p. 2.

${ }^{29}$ J. Updike, "The Mystery of Mickey Mouse ", [dans :] R. Atwan (dir.), The Best American Essays. College Edition, Boston, Houghton Mifflin Company, 1995, p. 390, trad. M.A.

${ }^{30} \mathrm{Cf}$. S. Bukatman, The Poetics of Slumberland: Animated Spirits and the Animating Spirit, op. cit. 
Chaplin pour exprimer des pensées qui sont officiellement interdites. Achtung Zelig! fonctionne d'une façon similaire en évoquant les mémoires médiatiques, y compris les traditions du fantastique et de l'absurde pour narrer I'indicible. Cet ancrage est signalé par la juxtaposition du visage d'Émile avec les postures burlesques de Chaplin, qui suggère qu'Émile se situe dans la filiation d'un genre distinctif de comédie inventé par Chaplin, où I'humanité persiste contre tout, même dans les situations les plus impossibles et les plus absurdes. Comme le soulignent les mouvement mécaniques, cette humanité est distinctivement moderne, mais enracinée dans le corps, sa grâce, ses mouvements burlesques et sa résilience. Cette comédie burlesque et chaplinesque est perceptible dans Achtung Zelig!, non seulement dans les actions déjà décrites, mais aussi dans les gestes pantomimiques des soldats allemands. Comme Émile, Hynkel est un personnage plus ridicule que terrifiant. Ses discours de haine et ses bouffées de rage sont des parodies incompréhensibles des discours d'Hitler qui retiennent le ton hystérique tout en effaçant leur pouvoir significatif (ce qui rappelle aussi Arturo Ui de Brecht).

"Nous pensons trop et sentons trop peu. Plus que des machines, nous avons besoin d'humanité. Plus que d'ingéniosité, nous avons besoin de la compassion et de la gentillesse ", dit le barbier dans la scène finale du Dictateur. Ces mots font partie du discours que le barbier, confondu avec le dictateur, délivre à la masse des partisans d'Hynkel. Parfaitement compréhensible, ce discours renverse le message de haine et de division d'Hynkel en un message d'empathie. II réduit ainsi la cause fondamentale du traumatisme, la destruction de tous les liens empathiques, comme le manifeste le soulagement sur le visage d'Hannah quand elle entend la voix de son amant. Le message du barbier résonne à travers l'espace onirique des forêts luxuriantes, les personnages et événements impossibles d'Achtung Zelig!. 
La BD est habituée aux êtres autrifiés, aux modes et aux mondes absurdes et fantastiques, comme le montre Achtung Zelig!. Par un rejet de la logique et l'évocation de l'absurde qui font écho aux films de Chaplin et à la BD de Rosenthal, les mémoires médiatiques activées par Achtung Zelig! vont bien au-delà du carnavalesque afin de mobiliser le rire et l'absurdité face à l'incompréhensibilité des événements traumatisants. 
Achtung Zelig! : deux monstres et un clown au carrefour de l'histoire et de la mémoire médiatique
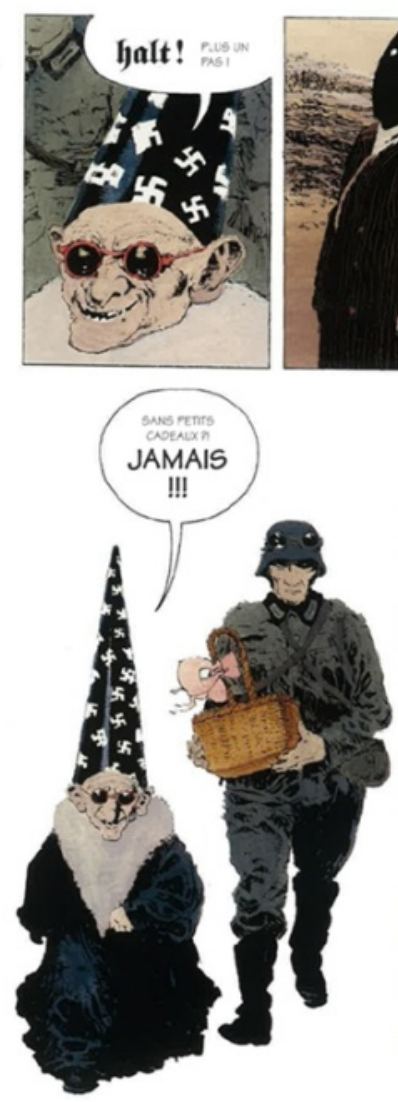
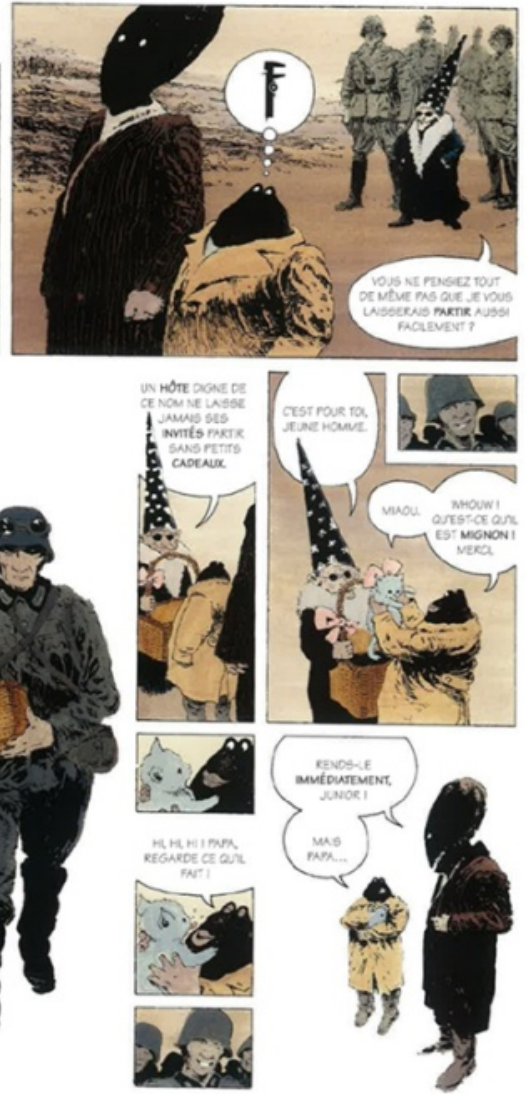

Fig. 1. Achtung Zelig!, p. 5. Krystian Rosenberg et Krzysztof Gawronkiewicz (C) Casterman. Avec l'aimable autorisation des auteurs et des Éditions Casterman 


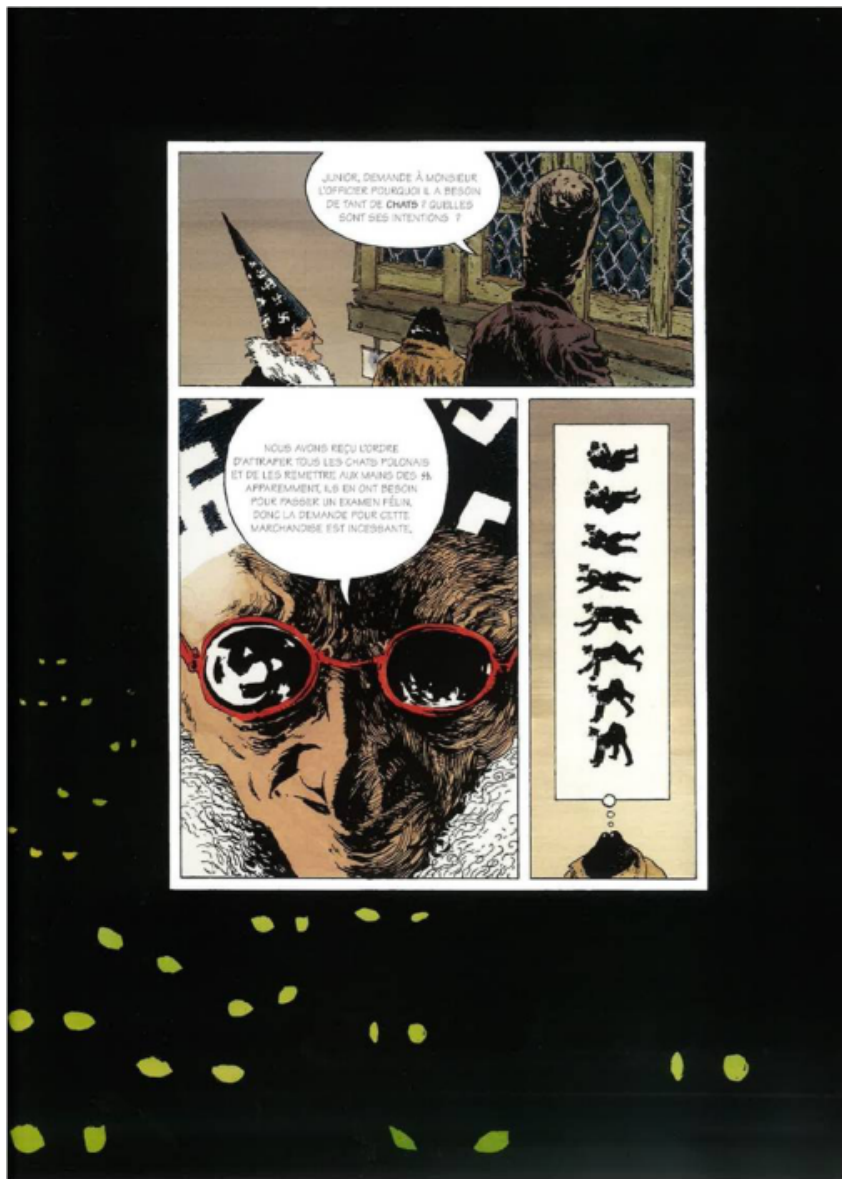

Fig. 2. Achtung Zelig!, p. 19. Krystian Rosenberg et Krzysztof Gawronkiewicz (C) Casterman. Avec l'aimable autorisation des auteurs et des Éditions Casterman 
Achtung Zelig! : deux monstres et un clown au carrefour de l'histoire et de la mémoire médiatique

\section{bibliographie}

Bakthin M., Rabelais and His World, H. Iswolsky (trad.), Bloomington, Indiana University Press, 1984.

Birek W., " Grafische Stilistik der Comics Krzysztof Gawronkiewiczs. Versuch einer Charakterisierung ", [dans :] K. Kupczynska, R. Makarska (dir.), Comic in Polen/Polen im Comic, Berlin, Christian A. Bachmann, 2016.

Bolter J., Grusin R., Remediation: Understanding New Media, Cambridge, MIT Press, 2000.

Bond L. et al., Memory on the Move: Tracing the Dynamics of Memory Studies, New York, Berghahn, 2017.

Bukatman S., The Poetics of Slumberland: Animated Spirits and the Animating Spirit, Berkeley, University of California Press, 2012.

Butler J., Gender Trouble: Feminism and the Subversion of Identity, London, New York, Routledge, 1990.

Carroll N., "Horror and Humor ", [dans :] The Journal of Aesthetic and Art Criticism, 1999, $\mathrm{n}^{\circ} 2$.

Cohen J., "Monster Culture (Seven Theses) ", [dans :] Idem (dir.), Monster Theory: Reading Culture, Minneapolis, University of Minnesota Press, 1996.

Erll A., Rigney A., "Introduction: Cultural Memory and its Dynamics ", [dans :] Idem (dir.), Mediation, Remediation and the Dynamics of Cultural Memory, Berlin, Walter de Gruyter, 2009.

Gawronkiewicz K., Rosenberg K., Achtung Zelig!, W. Kasprzak (trad.), Brussels, Casterman, 2004.

Huyssen A., Present Pasts: Urban Palimpsests and the Politics of Memory, Stanford, Stanford University Press, 2003.

Haudot J., Shoah et bande dessinée, Paris, L'Harmattan, 2012.

Jürgens A.-S., "The Joker: A Neo-Modern Clown of Violence ", [dans :] Journal of Graphic Novels and Comics, 2014, n० 4.

Kotek J., Pasamonik D., Mickey à Gurs : Les carnets de dessin de Horst Rosenthal, Paris, Calmann-Lévy, 2014.

LaCapra D., Writing Trauma, Writing History, Baltimore, John Hopkins University Press, 2000.

Morgan G., "Speaking the Unspeakable and Seeing the Unseeable: The Role of Fantastika in Visualising the Holocaust, or, More than just Maus ", [dans :] The Luminary, 2015, $n^{\circ} 6$, http://www.lancaster.ac.uk/luminary/ issue6/issue6article3.htm.

Neiger M. et al. (dir.), On Media Memory. Collective Memory in a New Media Age, Basingstoke, Palgrave Macmillan, 2011.

Ngai S., Our Aesthetic Categories: Zany, Cute, Interesting, Cambridge, Harvard University Press, 2012.

Rothberg M., " "We Were Talking Jewish": Art Spiegelman's Maus as "Holocaust" Production ", [dans :] Contemporary Literature, 1994, n 4. Round J., Gothic in Comics and Graphic Novels: A Critical Approach, Jefferson, McFarland, 2014.

Smolderen T., Naissance de la bande dessinée: De William Hogarth à Winsor McCay, Bruxelles, Les Impressions Nouvelles, 2009. 
Spiegelman A., The Complete Maus, London, Penguin Books, 2003.

Updike J., "The Mystery of Mickey Mouse ", [dans :] R. Atwan (dir.), The Best American Essays. College Edition, Boston, Houghton Mifflin Company, 1995.

Van Dijck J., Mediated Memories in the Digital Age, Stanford, Stanford University Press, 2007.

\section{abstract}

\section{Achtung Zelig!: Two Monsters and a Clown at the Crossroads of History and Media Memories}

Unfolding during the Second World War Achtung Zelig! adopts a mode of narration that is both fantastic and absurd. The comic not only reflects the issues surrounding the representation of the unimaginable and real horrors of the Holocaust but it also exemplifies the presence and functioning of 'media memories' or memories of specific cultural productions as well as media and the performing arts (circus, theatre). This article examines the functioning, particularly the generation of connotations, of the key media memories of the carnivalesque, Art Spiegelman's Maus, Horst Rosenthal's Mickey au camp de Gurs and Charlie Chaplin's The Great Dictator in Achtung Zelig!

\section{keywords}

comics/graphic novels, Holocaust, memory, carnivalesque

\section{mots clés}

BD/roman graphique, Holocauste, mémoire, carnavalesque

\section{maaheen ahmed}

Maaheen Ahmed est chargée de recherches du Fonds de la Recherche Scientifique - Flandre (FWO) à l'Université de Gand. En ce moment elle étudie la représentation des esprits et mémoires troublés dans les BD anglophones et francophones. Son premier ouvrage, Openness of Comics: Generating Meaning within Flexible Structures est paru aux Presses universitaires de Mississippi en 2016. Elle vient de terminer une deuxième monographie sur les inclinaisons romantiques des gentils monstres dans la BD. Elle a aussi dirigé, avec Stéphanie Delneste et JeanLouis Tilleuil, The Cultural Standing of Comics/Le statut culturel de la BD (Academia-L'Harmattan, 2017). 